\title{
Educación prenatal: factor asociado al éxito de la lactancia materna ${ }^{1}$
}

\author{
Zully Araya Cubero ${ }^{2}$ \\ Geovanna Brenes Campos ${ }^{3}$
}

Institución: Universidad de Costa Rica

Programa CIEBE-CR

\section{COMO CITAR}

Araya, Z. y Brenes, G. (2012). Educación prenatal: factor asociado al éxito de la lactancia materna. Rev. Enfermería Actual en Costa Rica, 23, 1-14 Recuperado de: <http://www.revenf.ucr.ac.cr/lactanciamaterna.pdf> ISSN 1409-4568

\section{RESUMEN}

El presente artículo tiene como objetivo investigar si la educación sobre lactancia materna (LM), durante el periodo prenatal, es un factor que contribuye al éxito de esta, en comparación con la brindada a la puérpera durante su estancia hospitalaria y el postparto. Con el fin de identificar la evidencia científica, se formuló una pregunta clínica en formato PICO. Luego, se utilizaron diversas bases de datos, entre ellas el buscador GOOGLE ACADÉMICO, PUBMED, MEDLINE, EBSCO y COCHRANE DATABASE para la recuperación de la evidencia. Se obtuvo 207 artículos de los cuales 12 proporcionaron datos que responden, parcialmente, a la interrogante planteada. La información fue sometida a análisis crítico, mediante la plantilla CASPe y AGREE, con el fin de determinar si era posible responder a la pregunta propuesta. Se concluye que existe evidencia que indica que las intervenciones para promover y apoyar la lactancia materna aumentan las tasas de iniciación, duración y exclusividad de esta. La promoción y el apoyo de la lactancia materna se puede lograr a través de intervenciones en el transcurso del embarazo, en el momento del parto, después de este y durante la lactancia; sin embargo, son necesarios más estudios controlados que indiquen si la información que reciben las mujeres sobre lactancia materna en la etapa prenatal, comparada con aquella que se les da solamente en la etapa postparto disminuye el riesgo de abandono de la lactancia materna.

Palabras claves: lactancia materna, educación prenatal y educación postparto.

\footnotetext{
${ }^{1}$ Fecha de recepción: 26 de agosto del $2012 \quad$ Fecha de aceptación: 17 de setiembre del 2012

${ }^{2}$ Enfermera Obstetra. CCSS, Área de Salud de San Ramón. Servicio de Enfermería. Correo Electrónico: zujdjp@hotmail.com

${ }^{3}$ Enfermera Docente, Escuela de Enfermería de la Universidad de Costa Rica. Correo Electrónico: johabreca105@gmail.com
} 


\title{
.
}

(5)

-

-

(-

\section{Revista Electrónica Enfermería Actual en costa Rica}

www.revenf.ucr.ac.cr

\section{Prenatal education: factor associated with the success of breastfeeding ${ }^{1}$}

\author{
Zully Araya Cubero ${ }^{2}$ \\ Geovanna Brenes Campos ${ }^{3}$
}

\begin{abstract}
Institution: University of Costa Rica
CIEBE-CR Program
\end{abstract}

\section{CITED}

Araya, Z. y Brenes, G. (2012). Prenatal education: factor associated with the success of breastfeeding. Rev. Enfermería Actual en Costa Rica, 23, 1-14 Available: <http://www.revenf.ucr.ac.cr/lactanciamaterna.pdf $>$ ISSN $1409-4568$

\begin{abstract}
This article to investigate whether breastfeeding education during the prenatal period is a factor that contributes to the success of breastfeeding compared to the education provided to the mothers of newborns during their hospital stay and after birth. In order to identify the scientific evidence, a clinical question was raised PICO format, then used several databases including ACADEMIC GOOGLE search engine, PUBMED, MEDLINE, EBSCO and the Cochrane Database in recovering evidence. 207 items were recovered, of these last 12 provided results partially answer this question. The information was subjected to critical analysis by CASPe template and AGREE to determine whether it was possible to answer the question raised. Conclusion: There is evidence that interventions to promote and support breastfeeding rates increase initiation, duration and exclusivity of breastfeeding. The promotion and support of breastfeeding can be achieved through interventions in the course of pregnancy, at delivery, postpartum and during lactation, but need more controlled studies to indicate whether the information they receive breastfeeding women in the prenatal stage, compared with only the information they receive in the postpartum stage reduces the risk of abandonment of breastfeeding.
\end{abstract}

Keywords: breastfeeding, pregnancy education, postpartum education

\footnotetext{
${ }^{1}$ Date of reception: August 26, 2012

Date of acceptance: september 17, 2012

${ }^{2}$ Obstetric Nurse. CCSS, Nursing Service of San Ramon. E-mail: zujdjp@,hotmail.com

${ }^{3}$ Professor of nursing, School of Nursing. University of Costa Rica. E-mail: johabreca105@gmail.com
} 


\section{INTRODUCCIÓN}

"La lactancia materna es una costumbre ligada a la supervivencia de la especie humana, sin embargo debido al uso de la lactancia artificial, factores sociales, económicos y culturales que rodean al individuo y su familia" (Díaz, Díaz, Fernández, 2006, p 1.), ha sufrido un abandono masivo en todo el mundo. Las organizaciones de salud a nivel global, regional y nacional desarrollan lineamientos, políticas y normativas ante la evidencia científica que manifiesta la superioridad de la lactancia materna como un factor positivo de la salud materno- infantil.

Tal como lo describe Suárez (2001)

La lactancia materna es la única fuente que suministra todos los nutrientes necesarios para el correcto desarrollo del niño en los primeros meses de vida. Así lo han reconocido tanto la Organización Mundial de la Salud (OMS) como los informes de sociedades científicas. Así también se menciona que los beneficios de la lactancia materna van desde los aspectos nutricionales e inmunitarios hasta los psicológicos e intelectuales. (p.5)

La lactancia prolongada también beneficia a las madres. Según la Asociación Española de Pediatría:

El amamantamiento prolongado disminuye el tiempo de hemorragia menstrual (a lo largo de la vida de la mujer), reduce el riesgo y la gravedad de diversas infecciones, mejora el vínculo entre la madre y el hijo, y la autoestima como mujer y como madre. Además, reduce el riesgo de fractura espinal y de cadera posmenopáusicas; incluso en aquellos casos de restricción de la dieta por atopia asociada, se ha visto que no afecta a la mineralización ósea. También disminuye el riesgo de cáncer de ovario. Asimismo, se observa un riesgo menor, de hasta un 4,6\%, de cáncer de mama que aparece antes de la menopausia por cada 12 meses de lactancia, según un metaanálisis reciente 
(datos de 52 estudios de 30 países: 50.302 mujeres con cáncer y 96.973 controles; efecto independiente de otros factores de riesgo: paridad, edad, menopausia o BMI). En una sociedad en la que cada mujer tiene pocos hijos, la lactancia prolongada puede ser un factor especialmente protector frente al cáncer de mama. (AEP, 2004, p.88).

Las recomendaciones de la OMS y la UNICEF (2010) para una alimentación infantil óptima, tal como se estipulan en la Estrategia Mundial son: "Lactancia materna exclusiva durante los primeros 6 meses de vida e iniciar la alimentación complementaria, adecuada y segura, a partir de los 6 meses de edad, manteniendo la lactancia materna hasta los dos años de edad o más" (OMS, 2010, p.4).

Las revisiones de estudios realizados por la OMS (2010) en países en desarrollo muestran que los niños que no reciben lactancia materna tienen una probabilidad de 6 a 10 veces mayor de morir durante los primeros meses de vida; esto en comparación con los niños que son alimentados con leche materna. Muchas de estos fallecimientos son causados por diarrea y neumonía, más frecuentes y graves en niños alimentados de manera artificial (con leche artificial comercial). (p.4)

La investigación denominada: Factores socioculturales y psicológicos vinculados a la lactancia materna exclusiva, llevada a cabo en Cuba en el 2011, explica que:

según cálculos realizados por la UNICEF, en la actualidad en los países económicamente en desventaja, mueren aproximadamente 1500000 lactantes al año por falta de una adecuada lactancia materna, por haber recibido alimentación con productos sustitutos de la leche materna. Las tasas de mortalidad infantil varían de un país a otro. En general las naciones pobres tienen cifras mucho más altas que las desarrolladas. La mayoría de las muertes relacionadas con la alimentación artificial se manifiestan por enfermedades diarreicas. Solo en Latinoamérica hay más de 500000 muertes anualmente en menores de 5 años como resultantes de infecciones intestinales (Broche, Sánchez, Rodríguez y Pérez, 2011, p.17). 
Debido al panorama de disminución de la LM,

“en el 2001 UNICEF junto con la OMS, y con base en evidencia científica ampliamente respaldada, emite la recomendación internacional de promover y garantizar condiciones para una lactancia materna exclusiva desde el instante del nacimiento y hasta los seis meses de edad.” (UNICEF, 2011, p.3).

El Código Internacional de Comercialización de Sucedáneos de la Leche Materna (1981), la Declaración de Innocenti sobre la Protección, Promoción y Apoyo a la Lactancia Materna (1990), así como la Iniciativa Hospitales Amigos de la Infancia (1991) han venido llamando la atención de los estados y la opinión pública a mundial sobre los beneficios de la lactancia materna, así como acerca de los riesgos y costos en los que incurren familias y estados que no la promueven, la protegen y la difunden.

De esta manera, la Caja Costarricense de Seguro Social ha realizado un Manual para la Implementación Clínica de la Lactancia Materna y Desarrollo en el escenario hospitalario, el cual menciona que:

En Costa Rica de acuerdo a la Encuesta de Hogares del Instituto Nacional de Estadísticas y Censos (INEC) 2006, la lactancia materna al nacer se lograba en un 96\%, lactancia materna exclusiva a los 6 meses de sólo 10\% y una lactancia materna hasta los 2 años de un 25 \%. Esto implica que existen diferentes factores que influyen en que no se logre cumplir los ideales con respecto a una alimentación sana y adecuada, lo que implica grandes retos de intervención por parte de todos los sectores del país (CCSS, 2012, p.10).

Por otra parte, en Costa Rica la lactancia materna se ha impulsado por medio de programas y proyectos a pesar de la carencia de una política pública sobre el tema. Sin embargo, para el 2009 se aprobó una política de lactancia materna. Para ello se tomó en cuenta las pautas que brindan organizaciones como la OMS, la UNICEF,

“el Alto Comisionado de las Naciones Unidas para los Refugiados (ACNUR), la Convención Internacional sobre los Derechos del Niño y la Estrategia Mundial sobre la Alimentación del Lactante y el Niño, así como por el Estado y entes públicos costarricenses" (UNICEF, et al., 2011, p.10). 
La Política Pública de Lactancia materna, en su punto 2 señala que:

las instituciones públicas y privadas deben velar por el cumplimiento de la normativa vigente (Guías Clínicas, Guías de Atención, Normas, Protocolos, entre otros) relacionada con las buenas prácticas de lactancia materna, en todos los escenarios donde se tenga contacto con los niños, las niñas, sus madres y las familias (Asamblea Legislativa, 1994, p.5).

En la actualidad, el quehacer enfermero debe hacer un uso consciente y reflexivo de las mejores pruebas disponibles en la toma de decisiones sobre la atención integral de las personas. En el caso de la mortalidad infantil, está comprobado científicamente que la lactancia materna disminuye el riesgo de complicaciones, de enfermedades o muertes durante el primer año de vida en los niños y niñas. Por esto último, en Costa Rica se han implementado una serie de estrategias que permiten a la madre y su familia conocer acerca del tema de la lactancia, sus implicaciones para el infante, la familia y la sociedad en general.

Según Carrasco (2004), "la Enfermería Basada en la Evidencia (EBE) propone un nuevo modelo para realizar la intervención, convierte un problema clínico en una pregunta y la evidencia se genera a partir de cuatro conceptos generales". (Carrasco, 2004, p.2).

La investigación
La

experiencia
Expectativas,

experiencia

del usuario
Recursos

disponibles

Por tal motivo, la EBE toma en cuenta tanto la experiencia de los clínicos como la opinión de los usuarios de los servicios de salud, quienes exigen mayor información por parte del personal de salud para emitir criterios con respecto a sus propios cuidados.

Con base en el concepto de EBE, surgió una pregunta clínica con el propósito de obtenerla mejor evidencia que dirija las acciones de intervención del personal de salud y que garantice el éxito de la lactancia materna. Para el análisis de dicha interrogación, se planteó el siguiente objetivo: Investigar si la educación sobre lactancia materna durante el periodo prenatal es un factor que contribuye al éxito de la lactancia materna en comparación con la educación brindada a la puérpera durante su estancia hospitalaria y el postparto. 
A continuación, se realiza un análisis detallado de la metodología empleada y los materiales utilizados para dar respuesta a la pregunta.

\section{MATERIALES Y MÉTODOS}

En el presente estudio, se aplicó la metodología de la práctica clínica basada en la evidencia. Esta se compone de las siguientes etapas:

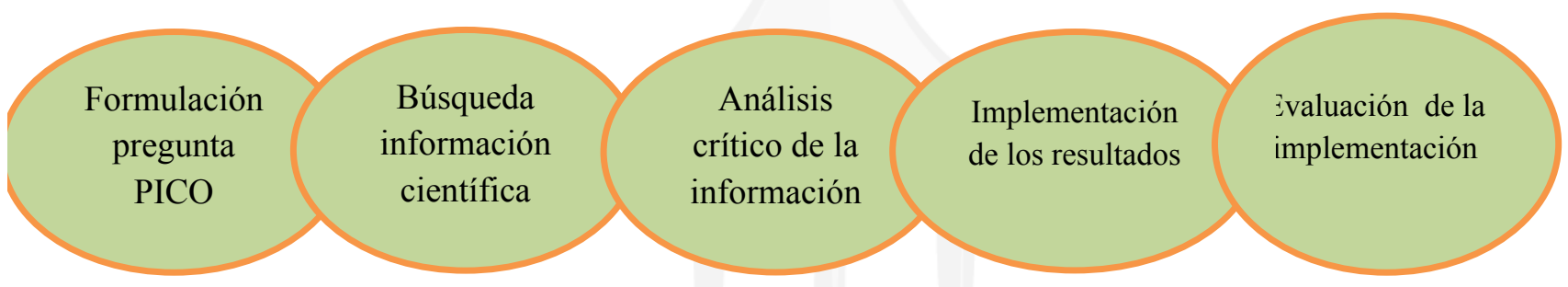

La pregunta en formato PICO se describe de la siguiente manera:

- El tipo de paciente o la patología de la que surge la pregunta $(\mathrm{P})$

- La intervención que se analizará. (I)

- La comparación con otra intervención (si procede) (C)

- Los resultados clínicos esperados $(\mathrm{O})$

Dada la inquietud de las investigadoras, surge la siguiente pregunta clínica:

En mujeres gestantes, ¿la información que reciben sobre lactancia materna en la etapa prenatal, comparada con la información que reciben solamente en la etapa postparto, disminuye el riesgo de abandono de la lactancia materna?

Para elaborar el estudio, se llevó a cabo una revisión bibliográfica de artículos científicos; esto con el fin de identificar la mejor evidencia relacionada con la pregunta clínica planteada, de manera tal que pueda ser contestada.

Se utilizaron varias bases de datos electrónicas, tales como el buscador GOOGLE ACADÉMICO, PUBMED, MEDLINE, EBSCO y COCHRANE DATABASE en la recuperación de la evidencia. Para la búsqueda bibliográfica, se utilizaron los descriptores: "breastfeeding or human lactation or breastfeed", en las bases en inglés, y "lactancia materna" en aquellas en español.

El resultado del proceso fue una base de datos con 207 artículos, 16 en resumen y 191 en texto completo. Únicamente 12 de ellos proporcionaron resultados pertinentes. De estos recursos, 1 corresponde a una guía de 
práctica clínica; 5 , a estudios cuantitativos; 2 , a estudios cualitativos; 1 , a un estudio cuali-cuantitativo y 3 , a revisiones sistemáticas.

Los instrumentos empleados para el análisis crítico de la evidencia recuperada fueron: el AGREE para Guías de Prácticas Clínicas, que evalúa tanto la calidad de la información aportada en el documento como la calidad de algunos aspectos de las recomendaciones (Coello, 2004) y el CASPe, un programa para ayudar a adquirir habilidades en el análisis y en lectura crítica de la literatura científica en salud (Lumbiganon, et al., 2011)

\section{RESULTADOS}

Los resultados obtenidos de los 12 documentos encontrados son producto de la aplicación de las plantillas CASPe según el tipo de investigación y AGREE para el caso de guías de práctica clínica. Estos se despliegan a continuación:

Hernández, et al. (2004) informan que,

existe evidencia que señala que la discusión en clases prenatales informales y con pocos participantes, lideradas por profesionales de la salud que enfaticen en los beneficios de la lactancia y den consejos prácticos, pueden aumentar las tasas de inicio de lactancia materna. Recomiendan, con categoría A, que el apoyo positivo y con conocimientos apropiados de la lactancia, por parte del personal sanitario, aumenta la incidencia, la duración y la exclusividad de la lactancia (p.27).

Según Hostalot, et al. (2001),

la información acerca de la LM que reciben las gestantes ha de basarse en criterios unificados. La puesta en marcha de protocolos conjuntos primaria-hospital y la creación de "Grupos de ayuda a la lactancia" facilita, para las madres, la decisión y el mantenimiento de esta (s.p.).

García, et al (2004) concluyen que "la prevalencia de lactancia materna aumenta en las mujeres que recibieron educación sanitaria y apoyo por parte del personal sanitario"(p 5)

En el 2001, en Prevalencia y duración de lactancia materna en Asturias. Suárez, Alonso, López, Martín, y Martínez concluyen que: 
Aunque en general las mujeres han sido seguidas durante el embarazo por más de un profesional, destaca el hecho de que haber sido atendida por la matrona constituye un predictor positivo para alimentar al niño de forma natural; es posible que las consultas de las matronas de atención primaria estén desarrollando una educación sanitaria individualizada y permitan resolver dudas respecto a la lactancia, algo que resultaría difícil que se produzca en otros entornos. Creemos que debe profundizarse en el papel que desempeñan los profesionales en la promoción de la lactancia natural, pues es en el transcurso del embarazo cuando un alto porcentaje de mujeres toma la decisión acerca del tipo de alimentación que dará a su hijo (p.108).

Con respecto a la educación brindada a las gestantes, Aparicio y Balaguer (2004) indican que el apoyo educativo a las madres en relación con la lactancia materna, tanto si se realiza antes como después del parto, mejora la proporción de madres que mantiene la lactancia materna exclusiva a los seis meses de vida del recién nacido. (p. 2)

Además, mencionan que, para conseguir esta mejoría, una educación postnatal de dos sesiones podría ser más efectiva que una única sesión prenatal. De esta manera, se refuerza la importancia de mantener educación continua con las madres durante los controles prenatales y no solamente en el periodo postparto.

Con base en el contexto de la madre, sus creencias y vivencias sobre la lactancia materna, en 2007 se realizó un estudio que concluye lo siguiente:

Se constata que la mayoría de las mujeres toman la decisión en el primer trimestre o antes, aunque muchas se deciden al final del embarazo y unas pocas después del parto. El estudio observa un aumento de dos veces la probabilidad de amamantamiento en los niños cuyas madres fueron aconsejadas por una matrona durante el embarazo. Sin embargo, otros autores refieren que el $50 \%$ de las madres entrevistadas refirieron haber decidido el tipo de alimentación después del parto, y 
creyeron que una entrevista pediátrica prenatal podría haber influido en su decisión (Llorens, Ferrer, Morales y Alenda, 2007, p. 5).

Además el mimo autor señala que,

Los programas de salud son la clave para fomentar la lactancia materna durante el embarazo y en los primeros meses tras el parto. Es fundamental la existencia de un entorno socio-sanitario adecuado que apoye a la mujer que opta por la lactancia como método de alimentación del recién nacido (Llorens et al, p.10).

En un estudio de carácter cuali-cuantitativo realizado en el 2007, se señala que las acciones de estimulo, protección y apoyo a la lactancia se sustentan en el reconocerla como un factor importante para mantener y desarrollar las estructuras económicas de un país e influir en la supervivencia del niño. El destete precoz coloca en riesgo la salud del infante y aumenta los índices de morbi-mortalidad infantil. Una de las causas indicadas para el destete precoz es la falta de conocimiento, por parte de la madre, acerca de la calidad de leche, tanto para el hambre del bebe como para el adecuado desarrollo de su hijo. Y menciona:

El conocimiento de las mujeres es un factor importante en el proceso de cambio de comportamiento, entre tanto este por sí solo, no garantiza el cambio de actitud. Las informaciones y las prácticas inadecuadas de los profesionales de la salud, también tienen influencia negativa para establecer y mantener la lactancia materna. Dentro de ellas se encuentra la falta de habilidad para apoyar a las madres que dan de lactar y al manejo clínico inadecuado de la lactancia materna (Beltrán, Ruiz, Grau, Álvarez, 2007,p.2).

Por su parte, Britton, McCormick, Renfrew, Wade \&King, (2008), en su revisión, indican que todavía no existen pruebas que evidencien que la duración de la lactancia materna mejore con el contacto prenatal habitual; 
se necesita de ensayos adicionales para evaluar la efectividad del apoyo profesional y no profesional en diferentes contextos.

Los ensayos deben considerar el momento y la administración de las intervenciones de apoyo, la efectividad relativa de los componentes de la intervención, opiniones de las mujeres, e investigaciones del entrenamiento más apropiado para las personas (profesionales o no profesionales) que proporcionan apoyo a las madres que amamantan.(p. 2)

Dyson, McCormick y Renfrew “establecen en las pruebas de su revisión que las distintas formas de educación sobre lactancia materna evaluadas resultaron eficaces para incrementar las tasas de inicio de esta entre las mujeres de ingresos bajos en los EE.UU” (2008, p.11). Sin embargo, la efectividad de las intervenciones revisadas debe evaluarse, en los diversos países y contextos, mediante estudios de poder estadístico adecuado que suministren detalles completos acerca del contenido de la educación impartida, de las personas a cargo de impartirla su entrenamiento y experiencia. Además, las intervenciones que combinan la educación sanitaria antes del nacimiento, junto con apoyo durante los días inmediatamente posteriores a este, deben evaluarse y compararse con aquellas que solo ofrecen educación.

Lumbiganon, et al. (2011), después de efectuar una revisión sistemática de artículos, concluyen que "hay una necesidad urgente de realizar un estudio controlado aleatorio de alta calidad, con un tamaño de muestra adecuado y que esté exento de influencias comerciales para evaluar la efectividad de la educación en lactancia materna." (p.10).

\section{DISCUSIÓN}

Existe evidencia que indica que las intervenciones lideradas por profesionales de la salud para la promoción y el apoyo de la lactancia materna aumentan las tasas de iniciación, duración y exclusividad de esta.

La evidencia destaca la atención por la matrona, la cual constituye un factor predictor positivo para alimentar al niño y la niña de forma natural. Es posible que las consultas de las matronas de atención primaria estén desarrollando una educación sanitaria individualizada y permitan resolver dudas respecto a la lactancia, lo que resultaría difícil que se produzca en otros entornos. 
El buen logro de la lactancia materna y la alimentación complementaria adecuada dependen de una información precisa y de un apoyo competente de la familia, la comunidad y del sistema de atención de salud. La Iniciativa Hospital Amigo del Niño (OMS/UNICEF) resume las mejores estrategias para la promoción, el apoyo y la protección de la lactancia materna. Es necesario reafirmar la importancia de que todas las instituciones públicas y privadas del país, que prestan servicios de salud para madres y niños/as, efectúen la totalidad de los diez pasos hacia una lactancia exitosa.

Se evidencia la necesidad de que el equipo de salud proporcione educación sobre LM, no solo a las futuras madres sino también a otros miembros de la familia; de esta forma, se garantiza el éxito de la lactancia para futuros hijos. La influencia positiva de la educación prenatal podría utilizarse para mejorar las condiciones de LM, pues es un recurso simple y de bajo costo.

La promoción y apoyo de la lactancia materna se puede lograr a través de intervenciones en el transcurso del embarazo, en el momento del parto, después del parto y la lactancia. En estas, se deben enfatizar en los beneficios de la lactancia y brindar consejos prácticos para aumentar las tasas de inicio de lactancia materna.

Es necesario realizar investigación primaria con estudios controlados acerca de la educación impartida y las personas a cargo de brindarla; esto para determinar si la información que reciben las mujeres acerca de LM durante la etapa prenatal, en comparación la que reciben solamente en la etapa postparto, disminuye el riesgo de abandono de la lactancia materna.

Se debe mencionar que esta investigación es una primera experiencia en la práctica de la Enfermería basada en la evidencia. Además, no se tuvo acceso a todas bases de datos; esto por el costo económico, lo cual dificultó la recuperación y revisión de la literatura de documentos completos. Las bases electrónicas de acceso abierto no contienen suficiente evidencia de estudios controlados ni guías clínicas que responda a la pregunta planteada.

\section{CONCLUSIÓN}

Existe evidencia que indica que las intervenciones de promoción y apoyo de la lactancia materna aumentan las tasas de iniciación, duración y exclusividad de esta. El objetivo se puede alcanzar a través de intervenciones en el transcurso del embarazo, en el momento del parto, después de este y la lactancia en marcha. Sin embargo, resulta necesario contar con más estudios controlados que indiquen si la información que reciben las mujeres acerca de LM, en la etapa prenatal, comparada con la información con la que cuentan solamente en la etapa postparto, disminuye el riesgo de abandono de la lactancia materna. 


\section{REFERENCIAS}

Aparicio, R y Balaguer, S. (2007). Con breves sesiones de educación de: sanitaria pueden aumentarse las tasas de lactancia materna exclusiva. Evidencia Pediátrica, 3:93, 335-596

Asamblea Legislativa. (1995). Regalmento a la Ley 7430. Gaceta_Nº174

Asamblea Legislativa.(1994). Ley 7430 Fomento a la Lactancia Materna. San Jose, Costa Rica.

Asociación Española de Pediatría. (2004) Guía para profesionales en Lactancia Materna. Recuperado de http://www.ministeriodesalud.go.cr/gestores en salud/lactancia/CNLM guia de lactancia materna_AEP.pdf.

Barrantes, R. (2000). Investigación: un camino al conocimiento, un enfoque cuantitativo y cualitativo. San José: EUNED.

Beltrán, B.; Ruiz, M.; Grau, R.; Álvarez, L. (2007). Efectividad de una estrategia de intervención educativa para la promoción de la salud con la embarazada. Revista cubana de salud pública, 33(2) 1-14

Britton, C.; McCormick, FM.; Renfrew, MJ.; Wade, A.; King, SE. (2008). Apoyo para la lactancia materna (Revisión Cochrane traducida). En: La Biblioteca Cochrane Plus, 2008 Número 4. Oxford: Update Software Ltd. Recuperado de http://www.update-software.com. (Traducida de The Cochrane Library, 2008 Issue 3. Chichester, UK: John Wiley \& Sons, Ltd.).

Broche, R; Sánchez, O y Rodríguez, D. (2011). Factores socioculturales y psicológicos vinculados a la lactancia materna exclusiva. Revista cubana de medicina general integrada. 27(2). Habana, Cuba.

Caja Costarricense de Seguro Social. (2012). Manual de implementación clinica de lactancia materna y desarrollo. Escenario Hospitalario. San José: CCSS.

Carrasco P. (2004) Importance of evidence-based nursing in the development of protocols and clinical guidelines. Recuperado de http://www.mednet.cl/link.cgi/Medwave/Enfermeria/junio2004/2750

Coello P., Ezquerro, O., Fargues, I., García, J., Marzo, M., Navarra, M., Pardo, J., Subirana, M. y Urrutia, G., (2004). Enfermería Basada en la Evidencia. Hacia la excelencia de los cuidados. Madrid: Ediciones DAE.

Delgado, N y Orellana, J. (2004). Conocimientos maternos sobre conductas óptimas de lactancia. Revista colombiana de obstetricia y ginecología, 55(1), 9-17.

Díaz, J; Díaz, M y Fernández, L. (2006). Educación prenatal y lactancia materna. Revista de salud cubana, 12 (9). Recuperado http://bvs.sld.cu/revistas/mciego/vol12_01_06/articulos/a9_v12_0106.html

Dyson, L; McCormick, F y Renfrew, MJ. (2008). Intervenciones para promover el inicio de la lactancia materna (Revisión Cochrane traducida). En: La Biblioteca Cochrane Plus, 2008 Número 4. Oxford: Update Software Ltd. Disponible en: http://www.update-software.com. (Traducida de The Cochrane Library, 2008 Issue 3. Chichester, UK: John Wiley \& Sons, Ltd.). 


\section{Revista Electrónica Enfermería Actual en costa Rica}

Palomares, M.J., Fabregat, E., Folch, S., Escrig, B., Escoín, F. y Gil C. (2011). Apoyo a la lactancia materna en una zona básica de salud, prevalencia y factores sociosanitarios relacionados. Revista Pediátrica de Atención Primaria. 13: 47-62.

Llorens, B.; Ferrer, E.; Morales, V.; Alenda, A. (2007). Creíamos en lactancia materna. Una aproximación de la experiencia en 3 generaciones. Index de Enfermería. 16 (58): 1-10.

Fondo de las Naciones Unidas para la Infancia (UNICEF). (2011). Análisis del modulo de lactancia Materna. Ministerio de Salud, Caja Costarricense del Seguro Social (CCSS), Instituto Nacional de Estadística y Censos (INEC). San José, Costa Rica.

García, M.C., García, S, Pi Juan, M., Ruiz, E. y Parellada, N. (2004). Lactancia materna: ¿puede el personal sanitario influir positivamente en su duración? Recuperado

http://www.elsevier.es/sites/default/files/elsevier/pdf/27/27v35n06a13073415pdf001.pdf

Grupo de trabajo CS-IHAN. (2009). Centros de salud IHAN (iniciativa de humanización de la atención al nacimiento y la lactancia). Una garantía de calidad. Revista pediátrica de atención primaria. 43 (11), 513-529.

Hernández, M, Cerveró, L, García, M, Fernández, M, Gutiérrez, G, Lloreth, J, Nebot, C, Segovia, C.(2004). Manejo de la Lactancia Materna desde el Embarazo hasta el segundo año. Guía de Práctica Clínica Basada en la Evidencia. España.

Hostalot, A.M., Sorní, A., Jovaní L., Rosal, J., Mercé, J., Iglesias J., Ariño, F., Castillo, T., López, M.J., Monllao, C., Querol, M.P., Arasa, M. y Ríos M. (2001). Lactancia Materna en el Sur de Cataluña. Recuperado de http://www.revespcardiol.org/sites/default/files/elsevier/pdf/37/37v54n03a10021548pdf001.pdf

Lumbiganon, P., Martis, R., Laopiboon, M., Festin, M., Ho, J. \& Hakimi, M. (2011). Educación prenatal en lactancia materna para aumentar su duración. Cochrane Database of Systematic Reviews DOI: 10.1002/14651858.CD006425

Ministerio de Salud. (2009). Politica Pública de Lactancia Materna. San José, Costa Rica.

OMS. (2010). Lactancia materna, cumplamos los 10 pasos. Recuperado de http://www.ministeriodesalud.go.cr/gestores_en_salud/lactancia/SML_WABA/SMLM_2012_folder_de_accion_para_web.p df.

Horta, B, Bahl, R, Martinez, J. \& Cesar, G. (2007) Evidence on the long-term effects of breastfeeding. Systematic reviews and meta-analysis. World Health Organization. Recuperado de http://whqlibdoc.who.int/publications/2007/9789241595230_eng.pdf

Hernández, M.T., Cerveró, L., García, M., Fernández, M., Gutiérrez, G., Lloret, J., Nebot, C. y Segovia, C. (2004). Guía de Práctica Clínica Basada en la Evidencia para el Manejo de la Lactancia Materna. En Pediatría Atención Primaria Área Recuperado de http://www.trainmed.com/trainmed2/contentFiles/10773/es/Guia_clinica_Lactancia_materna_2004.pdf

Organización Mundial de la Salud. (2010). La alimentación del lactante y del niño pequeño: Capítulo Modelo para libros de texto dirigidos a estudiantes de medicina y otras ciencias de la salud. Organización Mundial de la Salud. Sesión 4, 3038. Washington, D.C. 


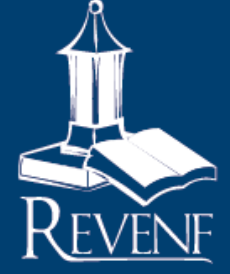

Spanó, A, Guerreiro, M, Bistafa, M. y Azevedo, F.(2007). El espacio social de mujeres y su referencia para el cuidado en la práctica de lactancia materna. Revista Latinoamérica Enfermagem, 15(2). Recuperado de htttp://www.eerp.usp.br/rlae.

Suárez, P., Alonso, J.C., López, A.J., Martín, D. y Martínez, M.M. (2001). Prevalencia y duración de lactancia materna en Asturias. Gaceta Sanitaria 2000, 15 (2), 104-110.

UNICEF, Ministerio de Salud, Caja Costarricense de Seguro Social y Instituto Nacional de Estadística y Censo (2011). Análisis del módulo de lactancia materna en Costa Rica 2011. Recuperado de: http://www.ministeriodesalud.go.cr/gestores_en_salud/lactancia/articulos/LAC informe lactancia_12122011.pdf 